\title{
How the Cycle of Scientific Knowledge is Reflected in the Course of Solid State Physics: the Effect of Magnetic Flux Quantization
}

\author{
N. Podoprygora ${ }^{1, *}$, A. Tkachenko ${ }^{2}$ \\ ${ }^{1}$ Department of Physics and Methodology of her Teaching, Kirovohrad Volodymyr Vynnychenko State Pedagogical University, \\ Kirovohrad, Ukraine \\ ${ }^{2}$ Department of Physics, Bohdan Khmelnytskyy National University, Cherkasy, Ukraine \\ *Corresponding author: npodoprygora@ukr.net
}

Received September 21, 2014; Revised October 30, 2014; Accepted December 14, 2014

\begin{abstract}
The article focuses on the practical realization of idea on presentation of cycle of scientific cognition in the process of arrangement theoretical problems of Physics on the example of study of Magnetic-Flux Quantization at a superconductor. The author examines the comparison of classic and quantum approaches in the process of theoretical explanation of Magnetic-Flux Quantization in Superconductors in a Solid State Physics.
\end{abstract}

Keywords: studies of Solid State Physics, integration of knowledge, principle of recurrence, superconductivity, Magnetic-Flux Quantization

Cite This Article: N. Podoprygora, and A. Tkachenko, "How the Cycle of Scientific Knowledge is Reflected in the Course of Solid State Physics: the Effect of Magnetic Flux Quantization." American Journal of Educational Research, vol. 2, no. 12B (2014): 61-69. doi: 10.12691/education-2-12B-12.

\section{Introduction}

Development of basic science and technological progress cause growth of humanity knowledge base, prompting scientists to search and develop new approaches in education and training to enable the assimilation not only of ready knowledge but leads to mastering new techniques studying. Teaching methods improvement aimed at individual personal development, free orientation within informational society, formation the universal abilities to overcome difficulties is an important today problem. Training activities in physics aimed at forming ability to think independently, to acquire and apply knowledge, properly consider and plan of operations, to seek solutions to the tasks, require implementation into the educational process of alternative forms and ways of life in which the significant role is assigned to research. Such activity aims at learner's cognition formation through investigation - inalienable quality of every person.

Under research activities, we understand such form of educational work that is related to solving those creative ones who study learners, research problems in various fields of science, technology and art. The concept of research in teaching physics, offered by psychologists M.G.Alekseev, O.V.Leontovich, S.O.Obuhov, emphasizes the differences between scientific and research activities. When designing research activity the basis of a model and research methodology is taken into account developed and adopted in science for the last few centuries. This model is characterized by the presence of several standard phases, presented at any scientific study, irrespective of the visual area in which it develops. Thus the main goal of educational research from the functional point of view is different from the one that exists in the field of science. If the main aim in science is to produce learner's new knowledge in the general cultural sense, thus the main aim in education lies in formation functional skills research as a universal way of reality understanding through providing motivation for training activities and personal position intensification of the subject study in educational process, based on a subjective gaining new knowledge (ie, independently obtained knowledge that is new and personally meaningful for the learners) [1]. Based on his own experience of research in physics, we found out that this type of activity is a developmental tool the learners motivation to learn and a major regulator of the quality of education [2,3]. Therefore, students' research activities are considered as one of the conceptual foundations of teaching physics. However, while teaching students physics there always be an urgent problem left in the formation of future professionals not only relevant subject competencies in the context of strengthening their professional training, but also from the point of their variable and critical thinking as to formation a scientific outlook of mankind. In our opinion, the most favorable conditions for solving the problem created while teaching students with the methodology of scientific knowledge based on the principle of teaching physics recurrence. That allows the teacher to engage students into research activities, to enrich their experience of its implementation 
and, at the same time, take care of ability conditions in educational information student's perceptions.

\section{Research Methodology}

Scientific method of cognition - is a tool for learner's cognitive development and creativity, it enables their independent thinking and encourages them to work. In our opinion, one of the main sources of teaching physics methods development and practice modernization is the methodology of teaching and learning activities, which is based on modern scientific methods of physical phenomena and processes studying.

\subsection{The Principle of Cycling}

In the methods of teaching physics at secondary school at Soviet Union in the 70's of last century B. Razumovsky was the first to offer universal methodological facility of academic knowledge organization - the principle of cyclic, submitted by the following logic of students' teaching and learning "facts problem - hypothesis, model - result experiment, practice" [4]. This idea turned out to be the most appropriate for us and has been implemented in practice-level objective while formating of students' functional research skills while solving experimental problems in physics [2] and in the series of scientific knowledge organization and implementation through a number of experimental tasks formulation (study of solids elastic properties, strain gage actions, the definition of the liquids surface tension) [3].

Cycles of teaching physics at high school we consider in the approximation methods of scientific knowledge on methods of teaching physics by presenting a comprehensive experimental and theoretical methods of physics to their subject-level [5]. Variant of uncertainty complex representations is offered and based on continuity and teaching physics cyclical at Pedagogical University. Specifically, we found that the idea of varied theoretical approaches comprehensive presentation to the use of uncertainties ratios in a series of basic courses, future teachers of physics professional training based on teaching physics cultural approach contributes to formation the mankind scientific outlook. However, the variability of ratios input methods of uncertainty is useful in applications to perform semi-quantitative microworld phenomena estimations, coordination of corpuscular and microparticles wave properties, determination of the applicability criteria for these concepts to classical mechanics. But the main thing is that the study in various ways Heisenberg's uncertainty relations and explanation of their contents allows the teacher to engage students to research activities to enrich their experience [6].

\subsection{Modeling}

Modeling is an essential step in any purposeful activity and takes the lead in modern scientific research. In pedagogy, as the V.I. Myheyev says "widespread ... modeling ... explains the diversity of its epistemological functions, which lead to educational phenomena and processes study at a special facility - a model that is intermediate between the subject - teacher, researcher and research subject, namely certain properties and relations between the educational process elements" [7].

The process of physics students teaching on the basis of scientific knowledge, we see in the use of different modeling features, reflected in its functions: cognitive - as the method of educational knowledge in the process of cognitive image of study object. In our opinion, the cognitive function implementation does not involve scientific knowledge process, its purpose lies in familiarizing students with the most rational way of physical process or phenomenon investigation; Heuristic as the means of efficient assimilation of new teaching material. In applied methods of teaching physics simulation oriented primarily on developing students' skills to carry out mathematical modeling of real physical processes and phenomena; Visual - the principle of modeling is the highest degree of visibility principle manifestation, its development and generalization connected with critical changes in the purposes and types of educational process; Integrative - as a means of systematization and generalization of knowledge, including mathematical models standardization of physical objects under study is a means of summarizing the studied physical phenomena and processes generalization; Activity - as the means of planning and management of training and search (research) activities; Developing - as the means of theoretical thinking, students' creative abilities, knowledge perception activation.

Taking into account all these features enables modeling, in our opinion, adequately plan and implement learning activities for students of physics consistency of the target, the semantic and procedural components of the suggested methods of teaching the discipline.

Mathematical modeling is the means of introducing students to the cognitive activity of Physics, aided content course in theoretical physics. It provides integration of physical and mathematical knowledge of students into the educational activities applied dimension. However, we realize that we must try to teach students theoretical physics relying on an experimental basis, which are both the basis and the criterion of theoretical research validity. Therefore, in our opinion, the principle of teaching physics recurrence, implemented under these scheme "empirical facts, the problem $\rightarrow$ hypothesis $\rightarrow$ mathematical model theoretical result $\rightarrow$ experimental verification, the possibility of practical implementation, the prospects for further research" on how to present a series of scientific knowledge in theoretical research in physics. This idea we were guided in the development of curricula and textbooks of theoretical physics $[8,9,10]$.

\section{Tasks of Investigation}

The scientific method of cognition is one of the human culture achievements that has come a long historical way of its developing and is the basis of cultural approach to teaching physics. Display logic cognition of theoretical physics research facility in the historical and genesis plan allows: display its physical meaning, the stages of research in science, scholars' controversial points of view while justifying its theoretical properties and manifestations; detecting imperfections of certain 
mathematical models, consistency and criteria devolution of relevant theoretical concepts and perspectives of further development etc.. Therefore, the main objective of our research is to present methodological development of object-practical implementation of the scientific knowledge presentation idea in theoretical studies based on the example of students' study of the quantization of magnetic flux in superconductors' effect in the course of Solid State Physics.

\section{Studying the Effect of Magnetic Flux Quantization}

\subsection{Program of Study}

Macroscopic effect of magnetic flux quantization is one of the key problem of "Superconductivity" in the course of Solid State Physics, which is offered to be studied on the following program: superconductivity discovery; superconducting state of substance critical parameters; Meissner's effect; magnetic flux quantization; London equation; Cooper pairs; coherence length; superconducting materials magnetic properties; prospects for superconductivity practical application [10].

In our opinion, before starting to study this effect, students should be aware of the physical meaning of the phenomenon and the condition of substance transition to the superconducting state. Information to the content of teaching material of historical information on this phenomenon discovery, experimental facts as to justifying its content, variation of classical and quantum approaches to the study of its mathematical models allows the presentation logic of scientific knowledge constructing a series in theoretical studies and students' current views formation on the physical outlook.

\subsection{The Main Material}

\subsubsection{Experimental Facts}

For the first time the effect of magnetic flux quantization in the superconducting ring with a current was foreseen by the German physicist F. London (1948). Later, his idea was by a Norwegian physical chemist L. Onsager at the International Conference on Theoretical Physics at Kyoto (Japan, 1953). The effect content is as follows: non-singly connected conductor (e.g., a ring) is placed in a magnetic field that induces a current in the conductor. At low temperatures (conductor is immersed in liquid helium), below the critical temperature Tc, at which the ring passes into the superconducting state and "captures" the external part of magnetic field, tat corresponds to discrete magnetic flux quantity:

$$
\Phi=\frac{h}{|2 e|} n=\Phi_{0} n(n=0,1,2,3, \ldots),
$$

where $h, e$ - fundamental physical constants: Planck's constant and the elementary charge. This superconducting current and the magnetic flux are stored indefinitely and during external field switching off, a structure is formed that resembles a magnet. The quantity $\Phi_{0}=h /|2 e|=2,067833636(81) \cdot 10^{-15} \mathrm{~Wb}$ is called the quantum of magnetic flux.
The quantity converted to the magnetic flux quantum $K_{J}=1 / \Phi_{0}=483597,9 \cdot 10^{9} \mathrm{~Hz} \cdot \mathrm{V}^{-1}$ is called Josephson constant.

It is believed that the superconducting current is a cumulative phenomenon, caused by particles (electron pair of oppositely directed spins) which are at the same state (phase coherence). Macroscopic discontinuity is connected to the fact that the electrons at the external environment changes turn to another quantum state not separately but all at once. Therefore, attempts of a theoretical reasoning of the magnetic flux quantization effect in superconductors continued long enough.

First, F. London believed that the quantity of the magnetic flux quantum was $\Phi_{0}=h / e$ (1948), as supposed that the superconducting current is formed by electrons. The necessity of formula adjustment for the quantum of magnetic flux calculating by replacing $e$ to $2 e$, that is, the idea that the quantum of magnetic flux can have a value $\Phi_{0}=h /|2 e|$, but $\Phi_{0}=h / e$ was expressed by L. Onsager (1959) at a conference on superconductivity at Cambridge (England) and later, after its experimental verification (1961) became accepted.

This assumption in the mathematical model of the superconducting current, which is formed by paired electrons with opposite spins $2 e$ and charge has been the basis of superconductivity microscopic theory BCS (1957), named after its developers, American physicists J. Bardeen, L. Cooper and D.R. Shrifera, for which they received the Nobel Prize in Physics in 1972. After the BCS theory creation, it became clear that the Londons' macroscopic theory formulas of superconductivity (1935), under the charge of current, which were identified with the charge of an electron $e$, is necessary to understand the Cooper pairs charge $|2 e|$.

In June 1961, two groups of experimenters - B. Deaver and W. Ferbenk (Stanford University, CA, USA) [11] R. Doll and M. Nebauer (Bavarian Academy of Sciences, Germany) [12] announced the discovery of the magnetic flux quantum. Experiments were only fine character. The magnetic field quantum itself was very small and it demanded work with weak magnetic fields and magnetic rings and conducting rings with a minimal area. To create such conductors Doll and Nebauer sprayed on a quartz string a thin layer of metal (lead). Consequently they got cylindrical conductor with 10 microns in diameter. This conductor hung perpendicular to another string of quartz and by its twisting while providing additional magnetic field it could be the observed trapped magnetic flux process. Deaver and Ferbenkom made like a tin vacuous cylinder about 2 microns in diameter, which was set in oscillating motion that induced currents in the surrounding registration system, the value of which depended on the trapped magnetic flux ring size. The experiments found out that the field, which was less than a certain amount isn't touched during its increasing one quantum of flux was touched which corresponded the value of $h /|2 e|$; during the next critical value exceeding two quanta flux were touched, etc., that fully confirmed the F.London's theoretical predictions with correction multiplier of $1 / 2$ for the magnetic flux quantum. At the same time it was the first BCS experimental confirmation, which foresaw magnetic flux quantization. 


\subsubsection{Theoretical Studies (Classical Approach)}

The attempt to build a macroscopic theory of superconductors, or rather their electrodynamics was carried out by brothers, German physicists Fritz and Heinz London (1935). Londons aimed to summarize the main experimental facts in mathematical form: the lack of Meissner effect resistance.

They supposed that the current carriers in superconductors, as well as in the metal, are the conduction electrons. Equal to zero resistance $(\rho=0)$ of superconductor means that the electrons during their motion are not subjected to collisions, i.e. accelerate under the influence of an electric field $\vec{E}$ freely:

$$
m_{e} \frac{d \overrightarrow{\mathrm{v}}}{d t}=e \vec{E}
$$

where $m_{e}$ and $e$ - mass and charge of the electron. This equation applies to all electrons. Therefore, it can be offered through a current density

$$
\vec{j}_{s}=n_{s} e \overrightarrow{\mathrm{v}}
$$

in:

$$
\frac{\partial \vec{j}_{s}}{\partial t}=\frac{n_{s} e^{2}}{m_{e}} \vec{E}=\frac{1}{\Lambda} \vec{E},
$$

where $\vec{j}_{s}$ - superconducting current density; $n_{s}$ - density of superconducting electrons; $\Lambda=m_{e} / n_{s} e^{2}-$ a constant. Equation (4), in its form - is the Newton's second law for superconducting electrons. It means that in a steady state, when $\partial \vec{j}_{s} / \partial t=0$, there isn't electric field in the superconductor. Here we did not consider the possibility of dimentional changes of the superconducting electrons chemical potential. This effect occurs for example in the superconductor near the border with ordinary metals, when current flows through this boundary.

We use one of Maxwell's equations, the physical meaning of which is associated with the electromagnetic induction phenomenon:

$$
\operatorname{rot} \vec{E}=-\frac{\partial \vec{B}}{\partial t}
$$

Taking the rotor from both sides of equation (4), taking into account (5), we obtain

$$
\frac{\partial}{\partial t} \operatorname{rot} \vec{j}_{s}=\frac{1}{\Lambda} \operatorname{rot} \vec{E}=-\frac{1}{\Lambda} \frac{\partial \vec{B}}{\partial t},
$$

or

$$
\frac{\partial}{\partial t}\left(\operatorname{rot} \vec{j}_{s}+\frac{\vec{B}}{\Lambda}\right)=0 .
$$

It means that the value in parentheses (7) remains:

$$
\operatorname{rot} \vec{j}_{s}+\frac{\vec{B}}{\Lambda}=\text { const. }
$$

But as the massive superconductor thickness is always $\vec{j}_{s}=0$ and $\vec{B}=0$, then $\operatorname{rot} \vec{j}_{S}+\vec{B} / \Lambda$ does not only persist, but always equals zero,

$$
\operatorname{rot} \vec{j}_{s}+\frac{\vec{B}}{\Lambda}=0
$$

Taking into account equation between the magnetic field and its intensity $\vec{H}, \vec{B}=\mu_{0} \vec{H}$, (9) it can be written differently:

$$
\operatorname{rot} \vec{j}_{s}+\frac{\mu_{0}}{\Lambda} \vec{H}=0
$$

Thus, the macroscopic theory of superconductivity is a simple theoretical attempt to describe the zero conductivity and Meissner effect in superconductor using London's phenomenological equations (4) i (9):

$$
\left\{\begin{array}{c}
\frac{\partial \vec{j}_{s}}{\partial t}=\frac{\vec{E}}{\Lambda} \\
\operatorname{rot} \vec{j}_{s}=-\frac{\vec{B}}{\Lambda}
\end{array}\right.
$$

These equations establish the link between electric current $\vec{j}_{s}$ and the magnitude of the electric $\vec{E}$ and magnetic field $\vec{B}$ in superconductors.

The system of Londons' equations (11) can be rewritten in a different way, the so-called aggregate basis, through the use of vector potential $\vec{A}$. Connection between $\vec{A}$ and $\vec{B}$ and $\vec{E}$ has been installed in classical electrodynamics and is as follows:

$$
\vec{B}=\operatorname{rot} \vec{A} ; \vec{E}=-\frac{\partial \vec{A}}{\partial t} .
$$

Then both system equations (11) can be written as one:

$$
\vec{j}_{s}=-\frac{n_{s} e^{2}}{m_{e}} \vec{A} \text { or } \vec{j}_{s}=-\frac{\vec{A}}{\Lambda} .
$$

\subsubsection{The Contradictions between Empirical Results and Implications of the Classical Londons' Theory}

The main reason for an alternative to the classical approach searching to describe the superconductivity phenomenon is the lack of mathematical model phenomenon. Let's try to explain this with on the example solution of the problem of the penetration problem of the magnetic field in a massive superconductor based on the Londons' theory according to Ersted law:

$$
\operatorname{rot} \vec{B}=\mu_{0} \vec{j}_{s} \text {. }
$$

Take the rotor from both sides of the equation

$$
\operatorname{rot} \operatorname{rot} \vec{B}=\mu_{0} \operatorname{rot} \vec{j}_{S}
$$

and take into consideration the second equation of the Londons' system (11),

$$
\operatorname{rot} \operatorname{rot} \vec{B}=-\frac{\mu_{0}}{\Lambda} \vec{B}
$$

Using the known information from mathematical theory of field relations for second-order differential operations

$$
\operatorname{rot} \operatorname{rot} \vec{B}=\operatorname{grad} \operatorname{div} \vec{B}-\Delta \vec{B},
$$

as well as the physical fact that magnetic charges do not exist, i.e. 


$$
\operatorname{div} \vec{B}=0
$$

obtain a differential equation for the magnetic field $\vec{B}$ in superconductors:

$$
\Delta \vec{B}-\frac{\mu_{0}}{\Lambda} \vec{B}=0, \text { or } \Delta \vec{B}-\frac{1}{\lambda_{L}^{2}} \vec{B}=0 .
$$

This second order differential equation of hyperbolic type, which is similar to equation-free sources of electromagnetic fields $\Delta \vec{E}-1 / c^{2} \vec{E}=0 \quad$ and $\Delta \vec{B}-1 / c^{2} \vec{B}=0$, but it instead of value $c^{2}$ - square of the circulation speed of electromagnetic waves in vacuum, appears another option

$$
\lambda_{L}^{2}=\frac{m_{e}}{\mu_{0} n_{s} e^{2}}, \text { or } \lambda_{L}=\sqrt{\frac{m_{e}}{\mu_{0} n_{s} e^{2}}},
$$

called Londons' penetration depth.

To estimate magnetic field depth of penetration in the superconductor we consider a simplified case. Let a superconductor occupies the half-space of an area $x 0 z$ $(0 z>0)$, and the magnetic field component $\vec{B}_{x}$ outside the superconductor is equal $\vec{B}_{0}$. Then, according to (19) for the magnetic field $\vec{B}_{x}$ components in the interior of the superconductor $0 z>0$ we get

$$
\frac{\partial^{2} \vec{B}_{x}}{\partial z^{2}}-\frac{1}{\lambda_{L}^{2}} \vec{B}_{x}=0,
$$

its solution is known from the differential equations general theory:

$$
\vec{B}_{x}=\vec{B}_{0} e^{-z / \lambda_{L}}
$$

so, the magnetic field at "e" times superconductor depth at the Londons' length $\lambda_{L}$ penetration. We evaluate magnetic field depth penetration into the superconductor. According to (20),

$$
\lambda_{L}=\sqrt{m_{e} / \mu_{0} n_{s} e^{2}} \approx 532 \stackrel{\mathrm{A}}{\mathrm{A}} .
$$

Thus, the magnetic field in the superconductor still penetrates, but a little depth. Experiments confirm this result for different substances $\lambda_{L}$ it is $400 \div 1000 \AA \mathrm{A}$. But the problem is: the depth penetration $\lambda_{L}$ also depends on temperature, approximately:

$$
\lambda_{L}(T)=\frac{\lambda_{L}(0)}{\sqrt{1-\left(T / T_{c}\right)^{4}}} .
$$

In the point $T_{c}$ superconducting transition $\lambda_{L}$ turns to endlessness.

Attempts to overcome the contradiction between empirical results and implications of the Londons' theory failed. This is due to the fact that superconductivity is the quantum phenomenon and therefore to explain all its experimental manifestations using classical laws fails.

\subsubsection{Theoretical Studies (Quantum Approach)}

In superconductors, like metal, conduction electrons carry electric current. However, significant differences between them do still exist. In metal electrons move noncorrelated under the influence of external electric fields (each on its own), in the superconductor a correlation electron motion appears. Part of the space within which electrons of superconductor "feel" each other is called the coherence length $\xi$.

Cause of the electrons motion correlation lies in the fact that in the superconductor there is an effective attraction between them. This attraction combines electrons with oppositely directed spins in pairs, called Cooper pairs. A typical size of such a pair is the coherence length $\xi$. In pure metals value $\xi \approx 10^{-6} \mathrm{~m}=10^{4} \stackrel{\circ}{\mathrm{A}}$. Not understandable remains the question: how such pairs can move in the metal without obstructing each other? According to Londons' penetration depth follows that the concentration of electrons in the metal, which take part in the superconducting current, the order $10^{28} \div 10^{29} \mathrm{~m}^{-3}$. This means that pairs penetrate one through another and the same time, like particles the electron gas, are free and do not interact with each other. This assumption is possible only from the quantum-mechanical point of view explaining the pass of Cooper pairs gas (similar to the electron gas) through the crystal lattice of the metal without spreading.

Let us find out the reason of the electrons attraction in a superconductor. Electrons are negatively charged particles and according to Coulomb's law, at first side, have repulse. But the Coulomb's repulsion occurs only in a vacuum, but in a superconductor (metal) electrons do not move in a vacuum, but in the vacant interstitial of crystal lattice. It is clear that during their movement they deform lattice. Deformation of lattices allows them to reduce the potential energy of the electrons to the place of deformation.

Consider a simple example to implement a didactic principle ability perception of educational information for students. Let the electron charge $e$, and the deformation of the crystal is near it $x$. Then the energy of electron interaction with the lattice deformation in general is a linear function of strain

$$
E_{\text {int }}=C e x,
$$

where $C-$ is proportionality sector that is constant of interaction. The deformation of the crystal lattice results in electron energy reduction. As $C>0$, energetically favorable compressive deformation with $x<0$, and when $C<0$ - tensile deformation, $x>0$. The total energy of the system "electron-lattice" $E_{\text {tot }}$ consists of electronic interaction power $E_{\text {int }}$, that decreases with increasing deformation and crystal lattice energy $E_{\text {lat }}$, which can be modeled as energy or tension of spring compression:

$$
E_{\text {lat }}=\frac{k x^{2}}{2},
$$

where $k-$ is the stiffness coefficient of the spring. Thus, the total energy of the "electron-lattice"

$$
E_{\mathrm{tot}}=E_{\mathrm{int}}+E_{\mathrm{lat}}=C e x+\frac{k x^{2}}{2} .
$$


The resulting deformation is determined on the condition of minimum total energy:

$$
\left.\frac{d E_{\mathrm{tot}}}{d x}\right|_{x_{0}}=\left.0 \Rightarrow(C e+k x)\right|_{x_{0}}=0 \Rightarrow x_{0}=-\frac{C e}{k} .
$$

Then the energy of "electron-lattice" at the point of minimum

$$
E_{\text {tot }}\left(x_{0}\right)=-\frac{C^{2} e^{2}}{2 k} .
$$

As can be seen, the minimum energy is proportional to the square of the constant interaction $C$, therefore, the result of the index $C$ does not depend. But at the same time, another thing is important: reducing the energy of the system due to the interaction is proportional to the charge square of the particle $e^{2}$. Therefore, when we consider two electrons, they're energetically favored to interact with one and the same deformation, not different.

Indeed, if each electron will cause a deformation, independently of each other, then the resulting reduction in energy systems "two electron-lattice" is twice as long, i.e. $2 E_{\text {tot }}\left(x_{0}\right)$. If two electrons are "combined" and will behave as one particle with charge $2 e$, then the gain in energy will be $4 E_{\text {tot }}\left(x_{0}\right)$, i.e. is twice more as electrons acted independently of each other. The difference between these two energies $\Delta E=2 E_{\text {tot }}\left(x_{0}\right)$ and the energy of attraction between electrons. If it exceeds the energy of repulsion, then Cooper pair is formed. Considerations being offered are rather schematic. We didn't take into account the fact that electrons move in a lattice (with sufficiently high speed). Nodes of crystal lattice fluctuate, etc. But a more correct consideration has a similar conclusion: the electron-lattice interaction always makes effective attraction between electrons. In some metals the effective attraction between electrons can prevail over their Coulomb repulsion, and this result into superconductivity. In other on the contrary - repulsion dominates and therefore these metals are not superconductors.

Such a mechanism of superconductivity is called phonon and was suggested in microscopic BCS theory of superconductivity. Phonon mechanism is called because the two electrons flying one past another, exchange virtual phonons (quantum of crystal lattice vibrations) with energy $\varepsilon_{\Phi}=\hbar \omega$ and impulse $\vec{p}_{\Phi}=\hbar \vec{k}$, as shown in Figure 1.

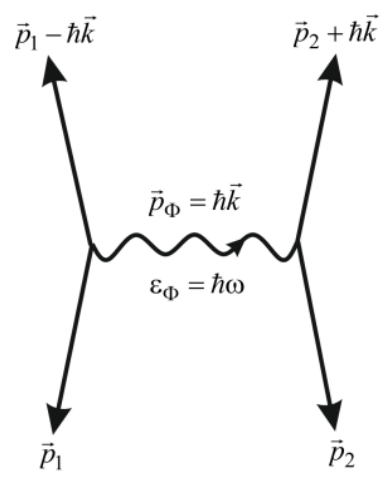

Figure 1. Feynman diagram: the interaction of two electrons of virtual phonons
Thus, the formation of Cooper pairs in a superconductor is energetically favored. Especially important is the fact that during the pairing of electrons in a Cooper pair, their spins are oppositely directed, so the total spin of the system equals to zero. Particles with integer or zero spin are called bosons, their behavior obeys quantum Bose-Einstein statistics. In the system of bosons Bose condensation takes place and the consequent manifestation of superfluidity similar to superfluidity of liquid helium phenomenon $\mathrm{He}^{4}$, which was discovered experimentally by Kapitza (1938) and theoretically grounded by Landau (1942). The main during its explanation is that liquid helium at extremely low temperatures behaves as a quantum liquid, quantum properties are able to express themselves at the macroscopic level.

The most important feature of quantum properties is that according to the concept of "quantum" a number of physical quantities characterizing quantum system, such as energy, impulse momentum, impulse moment projection on a random axis and so on can vary only discretely. For example, during the movement of the body in the usual viscous fluid friction, due to dissipation of energy, occurs at any speed of the body (even infinitely small). In quantum fluid everything is different. The dissipation of energy (and friction) is formed abruptly, provided that the body of certain critical speed. In a further slowing of the body friction for some limiting its value fully disappears. So superconductivity is superfluidity together with Cooper pairs ensembles - bose particles, like superfluidity of liquid helium $\mathrm{He}^{4}$, atoms of which are also bosons.

As Cooper pair formation is energetically favorable process, that's why to destroy a pair there's need to spend energy $\Delta \varepsilon_{s}$. Obviously, when the heat energy $k T$ compared with $\Delta \varepsilon_{s}$, Cooper pair is destroyed and superconductivity disappears. Therefore, $\Delta \varepsilon_{s} \approx k T_{c}$. If we consider the existence and speed of the electrons in the metal $-\mathrm{v}_{F}$ (speed of electrons having the Fermi energy), then coherence length can be defined as $\xi \approx \hbar \mathrm{v}_{F} / \Delta \varepsilon_{S}$. Speed Fermi for electrons conduction of metals is several units in $10^{5} \mathrm{~m} / \mathrm{s}$. Taking into consideration, for $\Delta \varepsilon_{s}$, critical temperature of the metal transition into the superconducting state we can choose such for mercury in the fundamental experiment of superconductivity detection, $T_{c}=4 \mathrm{~K}$ (1911), we get that the coherence length $\xi \approx 10^{4} \stackrel{\mathrm{A}}{\mathrm{A}}$, i.e. quantity, like we've obtained above.

\subsubsection{Matching Effects of Classical and Quantum Approaches}

Microscopic BCS theory is complex in the mathematical representation, but its ideas are very useful to justify some macroscopic manifestations of superconductivity, in magnetic flux particular quantum. With this aim, consider the features of the electromagnetic field modeling in terms of electrodynamics quantum.

The electromagnetic field of quantum ideas is examples of boson field. The intensity of its electric component $\vec{E}(\vec{r})$ can be regarded as the field amplitude. Energy 
density of the electromagnetic field $\varepsilon_{\mathrm{em}}$ - is a stream of photons, so the quasiclassical approximation:

$$
\varepsilon_{\mathrm{em}}=\varepsilon_{0}|\vec{E}(\vec{r})|^{2}=\varepsilon_{0} \vec{E} *(\vec{r}) \vec{E}(\vec{r}) \approx n(\vec{r}) \hbar \omega,
$$

where $n(\vec{r})$ - is a number of photons with frequency $\omega$, per unit volume; $\varepsilon_{0}$ - dielectric constant; $\vec{E}(\vec{r})$ - electric field amplitude, $\vec{E} *(\vec{r})$ - its conjugating quantity. Or, because of its magnetic induction component $\vec{B}(\vec{r})$ :

$$
\varepsilon_{\mathrm{em}}=\frac{|\vec{B}(\vec{r})|^{2}}{\mu_{0}}=\frac{1}{\mu_{0}} \vec{B} *(\vec{r}) \vec{B}(\vec{r}) \approx n(\vec{r}) \hbar \omega,
$$

where $\mu_{0}$ - magnetic constant; $\vec{B}(\vec{r})$ - magnetic field amplitude, $\vec{B} *(\vec{r})$ - its conjugating quantity. From classical electrodynamics we know that for the energy density of the electromagnetic field without its sources regard:

$$
\varepsilon_{\mathrm{em}}=\varepsilon_{0}|\vec{E}(\vec{r})|^{2}, \text { or } \varepsilon_{\mathrm{em}}=\frac{|\vec{B}(\vec{r})|^{2}}{\mu_{0}} .
$$

That's why during its calculation we can choose one of the components of the electromagnetic field $-\vec{E}(\vec{r})$ or $\vec{B}(\vec{r})$. Let's choose electrical component $\vec{E}(\vec{r})$.

Suppose that the total number of photons in the volume is more than one. Then for the amplitude field can be written:

$$
\left\{\begin{array}{l}
\vec{E}(\vec{r}) \approx\left(\frac{\hbar \omega}{\varepsilon_{0}}\right)^{1 / 2} n^{1 / 2}(\vec{r}) e^{i \theta(\vec{r})} \\
\vec{E} *(\vec{r}) \approx\left(\frac{\hbar \omega}{\varepsilon_{0}}\right)^{1 / 2} n^{1 / 2}(\vec{r}) e^{-i \theta(\vec{r})},
\end{array}\right.
$$

where $\theta(\vec{r})$ - phase field. It's necessary to take into account that from the quantum point of view, while mathematical modeling of electromagnetic fields as bosons flow, the amplitude of this field are amplitudes of probabilities. The quanta of such field are not photons, but Cooper pairs. Under these assumptions, the complete analogy with photons doesn't longer exist, but this analogy has proved to be very useful.

The main state of a superconductor is provided by weakly bounded Cooper pairs. Electron pair behaves like the boson, but each particular electron in pair is a fermion. Boson condensation temperature, which was calculated for the concentrations of electron charge, carriers in metals, equals Fermi temperature, i.e. $10^{4} \div 10^{5}$ K. The transition temperature into the superconducting state to normal is many times lower, $0,1 \div 10^{2} \mathrm{~K}$. Therefore they believe that the achievement of the transition temperature $T_{c}$ each electron pair decays into two fermions.

Model of superconductor as a system of non-interacting bosons should not be considered, as the volume per one Cooper pair, contains a large number of electrons order
$10^{6}$ microparticles. So, suggested considerations are applicable to the bose gas with a very large number of bosons in the same quantum state. Then the probability amplitudes for bosons can be interpreted as classical quantities like the photon flux of the electromagnetic field. But this approach is not justified for the metal in the normal state, as in this state the electrons are free and unpaired, and each electron behaves like a fermion.

Let's demonstrate that for the charged bose gas the given Londons' equation (13) comes true. Assume that the wave function $\psi(\vec{r})$ - is the probability amplitude for bosons. Let the concentration of these particles be constant, i.e.

$$
n_{s}=\psi(\vec{r}) * \psi(\vec{r})=\text { const. }
$$

For absolute zero bosons concentration temperature $n_{s}$ is twice less than the concentration of electrons in the conduction area, as considering bosons - are Cooper pair of electrons, then for the probability amplitudes can be written:

$$
\psi(\vec{r})=n_{s}^{1 / 2} e^{i \theta(\vec{r})}, \psi^{*}(\vec{r})=n_{s}^{1 / 2} e^{-i \theta(\vec{r})} .
$$

We note that the phase $\theta(\vec{r})$ wave function $\psi(\vec{r})$ is a very important quantity in our case. Comparing (32) and (34), a good enough approximation to the interpretation $\psi$ - functions as classical amplitude, but not as a quantum field operator. We present the speed of the particles in its quantum-mechanical coordinate image

$$
\hat{\overrightarrow{\mathrm{V}}}=\frac{1}{m_{e}}(\hat{\vec{p}}-q \hat{\vec{A}}(\vec{r}))=\frac{1}{m_{e}}(-i \hbar \vec{\nabla}-q \vec{A}(\vec{r})),
$$

where $q=|2 e|$ - is a charge of Cooper pair. Then, according to the classical formula for the density of electric current in the superconducting ring (multiply connected region), it can be given not only as $\vec{j}_{S}=n_{s} \overrightarrow{\mathrm{v}} q$, but in the quantum-mechanical representation, taking into account (33); (34) and (35), we'll get

$$
\vec{j}_{s}=\frac{q n_{s}}{m_{e}}(\hbar \vec{\nabla} \theta(\vec{r})-q \vec{A}(\vec{r})) .
$$

Taking the curl of both parts of this equation, we get:

$$
\begin{aligned}
& \operatorname{rot} \vec{j}_{s}=-\frac{n_{s} q^{2}}{m_{s}} \operatorname{rot} \vec{A}, \\
& \operatorname{or} \vec{j}_{s}=-\frac{n_{s} q^{2}}{m_{e}} \vec{A},
\end{aligned}
$$

i.e. the London equation (13) was necessary to prove. Mind that warning, for the final results identity was taken into consideration: $\operatorname{rot} \vec{\nabla}=[\vec{\nabla}, \vec{\nabla}] \equiv 0$.

Quantization of magnetic flux in a superconducting ring is a direct consequence of the relation (36). Let's consider the closed circuit $L$, located inside the superconductor. Let the circuit $L$ be located deep in the superconducting ring away from the surface (not to take into account the effect of the penetration of the magnetic field in the superconductor on the value of the penetration depth londonivskoy $\lambda_{L}$ ), as shown in Figure 2 . 


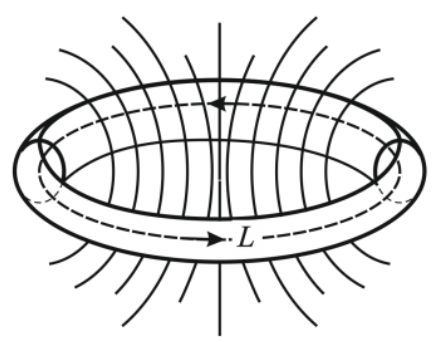

Figure 2. Circuit integration $L$ location in the superconducting ring with flux

According to the Meissner effect, the magnetic field inside the ring $\vec{B}$ and flux density $\vec{j}_{s}$ equal zero, so the left hand part (36) equals to zero:

$$
0=\frac{n_{s} q}{m_{e}}(\hbar \vec{\nabla} \theta(\vec{r})-q \vec{A}(\vec{r})) .
$$

The integrated form of this condition will have the following form:

$$
\oint_{L} \vec{\nabla} \theta(\vec{r}) d \vec{l}=\frac{q}{\hbar} \oint_{L} \vec{A}(\vec{r}) d \vec{l} .
$$

According to Stokes theorem the right side of the equation can be rewritten:

$$
\oint_{L} \vec{\nabla} \theta(\vec{r}) d \vec{l}=\frac{q}{\hbar} \int_{S} \operatorname{rot} \vec{A}(\vec{r}) d \vec{S} .
$$

As $\vec{B}=\operatorname{rot} \vec{A}(\vec{r})$, then

$$
\oint_{L} \vec{\nabla} \theta(\vec{r}) d \vec{l}=\frac{q}{\hbar} \int_{S} \vec{B}(\vec{r}) d \vec{S}
$$

Let's discover the circulation of the vector field $\vec{\nabla} \theta(\vec{r})$ along the closed contour $L$ towards its bypass $d \vec{l}$ :

$$
\oint_{L} \vec{\nabla} \theta d \vec{l}=\oint_{L} \frac{\partial}{\partial \vec{l}} \theta d \vec{l}=\int_{\theta_{1}}^{\theta_{2}} d \theta=\theta_{2}-\theta_{1},
$$

$\Delta \theta$ - is the phase change of the wave function of a single particle after passing circuit $L$. Probability amplitude for boson in the semiclassical approximation is the measured quantity, it's clearly defined, so,

$$
\theta_{2}-\theta_{1}=2 \pi n,
$$

where $n=0,1,2,3, \ldots-$ integer. The right part of condition (42) is the flow $\Phi$ of vector field $\vec{B}(\vec{r})$ through the surface $\vec{S}$, based on the circuit $\vec{L}$, considering its traversal (mnemonic rule right-hand screw rule) and a constant factor $q / \hbar$. Then, the final:

$$
2 \pi n=\frac{q}{\hbar} \Phi, \text { or } \Phi=\frac{2 \pi \hbar}{q} n,
$$

where $q=|2 e|$ - Cooper pairs charge; $\hbar=h / 2 \pi$ - Plank's reduced constant. This result is consistent with (1), i.e. the effect of quantization of magnetic flux in a superconducting ring.
In general, the total magnetic flux through the ring is the sum of the two fluxes: $\Phi_{\text {ext }}-$ from the external sources and $\Phi_{s}$ - from superconducting current flowing in the ring: $\Phi=\Phi_{\text {ext }}+\Phi_{s}$. Current $\Phi$ is quantized as a whole; but the flow of external $\Phi_{\text {ext }}$ any conditions quantization do not impose, while the flow $\Phi_{s}$ should "selforganize" so the full flow $\Phi$ had only a quantum quantity.

\section{Conclusion}

Methodological aspects of physics analysis in its historical development indicate its value the cultural, educational and developing potential. Organization of students' research activity in physics should include the value attitude formation not only to scientific discoveries, but also to the method of scientific knowledge, research methods, the science methodology in general. Systematization of scientific discoveries promotes scientific world reflection and scientific outlook formation. The scientific method of cognition and methods of natural phenomena in the content of education orientate students to independent learning activities and give them the opportunity to develop their cognitive and creative abilities. In particular, the comparative approach of classical and quantum mathematical describing models of magnetic flux macroscopic quantum effect enables the presentation of scientific knowledge logic in physics theoretical research as to students' modern scientific outlook in the given problem research activities.

Implementing cognitive, heuristic, visual, integrative, active and developmental functions of students' process modeling and performing research activities on theoretical physics is one of the methodological foundations for formation an open methodical system of physics teaching mathematical methods at universities.

\section{Acknowledgement}

We would like to thank the authors of the references who have helped us indirectly through their immortal books, journals while preparing this manuscript.

\section{Statement of Competing Interests}

The authors have no competing interests.

\section{References}

[1] Alekseev, N.G., Leontovich, N.G., Obuhov, S.A. and Fomina, L.F., "Conception of development of research activity of student (fragments)," Physics: teaching problems, 5. 3-5. 2006.

[2] Podoprygora, N.V., "Forming of functional research skills is during untiing of experimental tasks," Physics and astronomy is at modern school, 4. 11-15. 2013.

[3] Podoprygora, N., "Organization and realization of the experimental cycle of scientific cognition at Physics study," LatinAmerican Journal of Physics Education, 8 (1). 13-21. March. 2014 URL: http://www.lajpe.org.

[4] Razumovskiy, V.G., Development of creative capabilities student in the process of teaching physics. Inlightening, Moscow, 1975, 272. 
[5] Podoprygora, N.V., "About the studies of experimental and theoretical methods of physics in a pedagogical university" Scientific messages. Series: problems of method of physical and mathematical and technological education, 4 (1). 204-209 2013.

[6] Podoprygora, N. "Complex presentation of indeterminacy relation at educating of future physics teachers" in International Conference on Pedagogy of the 21st century: teaching in a world of constant information flow: Budapest. 29th-31st of August 2014, Society for Cultural and Scientific Progress in Central and Eastern Europe. Retrieved from mozila firefox on September 1, 2014. URL:

http://scaspee.com/6/post/2014/08/pedagogy-of-the-21st-centuryteaching-in-a-world-of-constant-information-flow.html.

[7] Miheev, V.I., A design and methods of theory of measurings is in pedagogics, KomKniha, Moscow, 2006, 200.
[8] Podoprygora, N.V., Sadoviy, M.I. and Tryfonova, O.M., The Mathematical methods of physics, Kirovohrad Volodymyr Vynnychenko State Pedagogical University, Kirovohrad, 2012, 300.

[9] Volchanskiy, O.V., Podoprygora, N.V. and Gur'evska, O.M., Thermodynamics and statistical physics, Kirovohrad Volodymyr Vynnychenko State Pedagogical University, Kirovohrad, 2012, 428.

[10] Podoprygora, N.V., Sadoviy, M.I. and Tryfonova, O.M., Solid State Physics, Kirovohrad Volodymyr Vynnychenko State Pedagogical University, Kirovohrad, 2013, 416.

[11] Deaver, B. and Fairbank, W. "Experimental Evidence for Quantized Flux in Superconducting Cylinders," Physical Review Letters, 7 (2).43-46. July. 1961.

[12] Doll, R. und Näbauer, M. "Experimental Proof of Magnetic Flux Quantization in a Superconducting Ring," Physical Review Letters, 7 (2). 51-52. July. 1961. 